International Journal of Modern Physics E

Vol. 20, No. 8 (2011) 1677-1686

(C) World Scientific Publishing Company

DOI: $10.1142 / \mathrm{S} 0218301311019659$

\title{
PARITY OF CHARGED PIONS
}

\author{
B. A. ROBSON \\ Department of Theoretical Physics, Research School of Physics and Engineering, \\ The Australian National University, Canberra ACT 0200, Australia \\ brian.robson@anu.edu.au
}

Accepted 14 February 2011

\begin{abstract}
This paper discusses the parity of charged pions. It is shown that the original determination of the parity of the negatively charged pion using the capture of negatively charged pions in deuterium to form two neutrons is not conclusive if the pion has a complex substructure. It is demonstrated that this experiment is compatible with the mixed-parity nature of the $\pi^{-}$arising in a recent composite model. A major consequence of this is that $\mathrm{CP}$ may be conserved in the $K^{0}-\bar{K}^{0}$ system.
\end{abstract}

Keywords: Standard Model; generation model; parity; CP violation; charged pions; neutral kaon decay; composite models.

PACS Number(s): 11.30.Er, 12.60.Rc, 13.25.Es, 14.40.Be

\section{Introduction}

The parity of charged pions has played a significant role in the overthrow of both parity $(\mathrm{P})$ conservation $^{1}$ and combined charge-conjugation-parity $(\mathrm{CP})$ conservation. ${ }^{2}$ In this paper we shall indicate how an early determination of the parity of the negatively charged pion led to the acceptance of both $\mathrm{P}$ violation in weak interactions and $\mathrm{CP}$ violation in the $K^{0}-\bar{K}^{0}$ system. We shall also show that recent substructure models of the charged pions and nucleons suggest that this early determination of the parity of charged pions was in principle inconclusive. A major consequence of this is that CP may be conserved in the $K^{0}-\bar{K}^{0}$ system.

In the Standard $\mathrm{Model}^{3}$ (SM) of particle physics, the parity of charged pions is assumed to be $P_{\pi}=-1$. This value was first obtained in 1954, within the theoretical framework of the time, by Chinowsky and Steinberger ${ }^{4}$ using the capture of negatively charged pions by deuterium to form two neutrons: $\pi^{-}+D \rightarrow 2 n$. This was prior to the quark model ${ }^{5,6}$ of mesons and baryons so that in the analysis of the experiment, the pion, the proton and the neutron were each assumed to be fundamental particles with no substructure.

Following the adoption of the quark model as part of the SM, the parity of the charged pions remained accepted as $P_{\pi}=-1$ : in the quark model, the $\pi^{-}$was 
proposed to be a combination of a down quark $(d)$ and an up antiquark $(\bar{u})$, i.e. $\pi^{-} \equiv$ $[d \bar{u}]$, so assuming the fundamental quarks have $P_{q}=+1$ while their fundamental antiquarks have $P_{\bar{q}}=-1$, the charged pions have $P_{\pi}=-1$. This follows since parity is a multiplicative operator in quantum mechanics and consequently $P[d \bar{u}]=$ $P_{d} P_{\bar{u}}[d \bar{u}]=-[d \bar{u}]$.

In Sec. 2. we shall examine in more detail the acceptance of $P_{\pi}=-1$ as the parity of the charged pions and provide a brief historical overview ${ }^{7}$ of how this value for the parity led to the discovery of $\mathrm{P}$ violation in weak interactions and also to the acceptance of CP violation in the $K^{0}-\bar{K}^{0}$ system.

In Sec. 3 we present a short description of both the Generation $\operatorname{Model}^{8-10}$ (GM) and a recent Composite Generation Model ${ }^{11,12}$ (CGM), which indicate that the parity of charged pions is not pure $P_{\pi}=-1$ but is a mixed-parity state, having a small $\left(\approx 5 \%\right.$ ) component of $P_{\pi}=+1$ parity. It has been shown, ${ }^{13}$ within the framework of the CGM, that the mixed-parity state of charged pions leads to a description of the 1964 observations of Christenson et al. ${ }^{2}$ without CP violation. It will be demonstrated that the experiment of Chinowsky and Steinberger is compatible with the mixed-parity nature of the $\pi^{-}$arising in the CGM.

Section 4 discusses some consequences of the mixed-parity quark states and states the conclusions.

\section{Parity of Charged Pions in the Standard Model}

In 1924 Laporte $^{14}$ analyzed the electromagnetic spectrum of iron and found two kinds of iron energy levels. He observed that transitions always occurred between the two kinds of energy levels and never between energy levels of the same kind. This selection rule, later known as Laporte's rule, was explained by Wigner ${ }^{15}$ in 1927. He demonstrated that both the two kinds of energy levels and the selection rule followed from the invariance of the Schrödinger equation under the operation of inversion of space coordinates.

This inversion operation became known as the "parity operator" in quantum mechanics and atomic states had either positive $(P=+1)$ or negative $(P=-1)$ parity depending whether the state was even or odd under the parity operation.

The two kinds of energy levels found by Laporte are states of positive and negative parity. The dominant electric dipole transitions between states of the same parity are forbidden by parity conservation, since the intrinsic parity of the emitted photon is negative. ${ }^{16}$ Thus Laporte's rule depended upon the conservation of parity for the electromagnetic interaction: nature appeared to exhibit complete symmetry under the inversion of space coordinates. Until 1957, this symmetry was held to be true for all physical processes.

In 1954 Chinowsky and Steinberger established, ${ }^{4}$ within the theoretical framework of the time, that the relative parity of the negatively charged pion and the nucleon was odd: if the nucleon had parity $P_{N}=+1$ then the $\pi^{-}$had parity $P_{\pi}=-1$. They employed the reaction involving the capture of negatively charged 
pions by deuterons to form two neutrons: $\pi^{-}+D \rightarrow 2 n$, a reaction suggested by Ferretti. ${ }^{17}$

The spins of the pion, the deuteron and the neutron were known to be 0,1 and $\frac{1}{2}$, respectively. It was also known that the pion is captured by the deuteron from an $\mathrm{S}$ state ${ }^{18}$ so that the relative orbital angular momentum in the initial state is $l_{i}=0$ and thus the total angular momentum of the initial state is $j_{i}=1$. An underlying assumption in the analysis was that the pion had a definite intrinsic parity: $P_{\pi}=+1$ or $P_{\pi}=-1$.

Thus, since the intrinsic parity of the deuteron was known to be $P_{D}=+1$ (this assumes that the neutron and proton have the same intrinsic parity), the parity of the initial state is given by

$$
(-1)^{l_{i}} P_{\pi} P_{D}=(-1)^{0} P_{\pi} P_{D}=P_{\pi} .
$$

The parity of the final state for relative orbital angular momentum $l_{f}$ is

$$
P_{n} P_{n}(-1)^{l_{f}}=(-1)^{l_{f}},
$$

assuming that the neutron has definite intrinsic parity $P_{n}=+1$ or $P_{n}=-1$. Consequently if parity is conserved in the reaction:

$$
P_{\pi}=(-1)^{l_{f}} .
$$

Since the neutrons are identical fermions, the allowed antisymmetric states $\left({ }^{2 S+1} L_{J}\right)$ of the two neutrons are ${ }^{1} S_{0},{ }^{3} P_{0,1,2},{ }^{1} D_{2},{ }^{3} F_{2,3,4}$, etc. The only final state with $j_{f}=j_{i}=1$ is the ${ }^{3} P_{1}$ state, so that $l_{f}=1$ and hence $P_{\pi}=-1$.

This value of the parity of charged pions led to the overthrow of both parity conservation (in 1957) and CP conservation (in 1964) in weak interactions. We now briefly describe how this came about.

In the period 1947-1953 several new particles with mass intermediate between the pion and the proton were discovered. In particular one charged meson, named the tau meson, decayed to three charged pions: $\tau^{+} \rightarrow \pi^{+}+\pi^{+}+\pi^{-}$, while another charged meson, named the theta meson, decayed to two pions: $\theta^{+} \rightarrow \pi^{+}+\pi^{0}$. Analyses $^{19-21}$ of the decays of both these particles indicated that they had closely identical masses and lifetimes. These properties suggested that the $\tau^{+}$and $\theta^{+}$ were simply two different decay modes of the same particle. However, evidence was accumulating that the tau and theta mesons were different particles.

In 1953-1954 Dalitz ${ }^{22,23}$ suggested that a study of the energy distribution of the three pions in tau meson decays would provide information about the spin and parity of the tau meson. Subsequent such analyses, ${ }^{24}$ assuming a charged pion parity $P_{\pi}=-1$, led to the conclusion that the tau meson had parity $P_{\tau}=-1$ and spin 0 or possibly 2 . On the other hand, the limited evidence ${ }^{25}$ available at the time suggested that the neutral pion had the same parity as the charged pions so that the theta meson had $P_{\theta}=+1$, if its spin was 0 or 2 , thereby violating the notion that the tau and theta mesons were the same particle. 
This tau-theta puzzle was resolved in 1956 by Lee and Yang, who suggested ${ }^{1}$ that perhaps parity was not conserved in the weak interaction processes involved in the two meson decays. This suggestion was rapidly confirmed. ${ }^{26-28}$ These $\mathrm{P}$ violating experiments indicated that the tau and theta mesons were indeed the same meson (later named $K^{+}$) with different decay modes and also showed that the weak interactions were not invariant under charge-conjugation $(\mathrm{C})$.

The discovery of parity violation implied that the weak interaction consisted of two terms with opposite parities. In 1958 it was shown ${ }^{29,30}$ that the so-called "V-A" theory of the weak interactions described the observed parity violations.

In 1947 a new neutral particle of similar mass as the $K^{+}$meson was discovered using a cloud chamber exposed to cosmic rays. ${ }^{31}$ This particle was initially called a V-particle because upon decay it displayed two tracks corresponding to charged particles forming a $\mathrm{V}$. The particle was a neutral $K$-meson (kaon) decaying to a $\pi^{+}$and a $\pi^{-}$meson. In 1950, an event was found ${ }^{32}$ in which the positively charged track appeared to be a proton: this was the first evidence of a $\Lambda^{0}$ hyperon decaying into a proton and a $\pi^{-}$meson.

By 1953, the Brookhaven Cosmotron was producing a significant number of such $\mathrm{V}$-particles and experiments ${ }^{33}$ demonstrated that the $\mathrm{V}$-particles were produced only in pairs, a typical reaction being:

$$
\pi^{-}+p^{+} \rightarrow \Lambda^{0}+K^{0}
$$

On the other hand, the $\mathrm{V}$-particles decayed individually in about $10^{-10} \mathrm{~s}$, which is about $10^{12}$ times longer than expected if the production process and decay mechanism are governed by the same interaction.

This paradox of the strange $\mathrm{V}$-particles was resolved by the introduction ${ }^{34,35}$ of a new additive quantum number called strangeness $(S)$, which was assumed to be conserved in strong interactions but not necessarily so in weak decay processes. The $\Lambda^{0}$ hyperon was allotted $S=-1$ while the $K^{0}$ meson was allotted $S=+1$. The non-strange particles, such as the charged pions, were allotted $S=0$. Thus the decay

$$
K^{0} \rightarrow \pi^{+}+\pi^{-}
$$

does not conserve strangeness and proceeds only via a weak interaction process, while reaction (4) conserves strangeness and proceeds via a strong interaction process.

In 1955 Gell-Mann and Pais ${ }^{36}$ considered the behavior of neutral particles under the charge-conjugation operator C. In particular they considered the $K^{0}$ meson and realized that unlike the photon and the neutral pion, which transform into themselves under the $\mathrm{C}$ operator so that they are their own antiparticles, the antiparticle of the $K^{0}$ meson (strangeness $S=+1$ ), $\bar{K}^{0}$, was a distinct particle, since it had a different strangeness quantum number $(S=-1)$. They concluded that the two neutral mesons, $K^{0}$ and $\bar{K}^{0}$, are degenerate particles that exhibit unusual 
properties, since they can transform into each other via weak interactions such as

$$
K^{0} \rightleftharpoons \pi^{+} \pi^{-} \rightleftharpoons \bar{K}^{0} \text {. }
$$

In order to treat this novel situation, Gell-Mann and Pais suggested that it was more convenient to employ different particle states, rather than $K^{0}$ and $\bar{K}^{0}$, to describe neutral kaon decay. They suggested the following representative states:

$$
K_{1}^{0}=\left(K^{0}+\bar{K}^{0}\right) / \sqrt{2}, \quad K_{2}^{0}=\left(K^{0}-\bar{K}^{0}\right) / \sqrt{2},
$$

and concluded that these particle states must have different decay modes and lifetimes. In particular they concluded that $K_{1}^{0}$ could decay to two charged pions, while $K_{2}^{0}$ would have a longer lifetime and more complex decay modes. This conclusion was based upon the conservation of $\mathrm{C}$ in the weak interaction processes: both $K_{1}^{0}$ and the $\pi^{+} \pi^{-}$system are even (i.e. $C=+1$ ) under the $\mathrm{C}$ operation.

The particle-mixing theory of Gell-Mann and Pais was confirmed in 1957 by experiment, ${ }^{37}$ in spite of the incorrect assumption of $\mathrm{C}$ invariance in weak interaction processes (C violation was discovered in 1957). The particle-mixing theory led to a suggestion by Landau ${ }^{38}$ that the weak interactions may be invariant under the combined operation $\mathrm{CP}$, although both $\mathrm{C}$ and $\mathrm{P}$ are individually maximally violated.

Landau's suggestion implied that the Gell-Mann-Pais model of neutral kaons would still apply if the states, $K_{1}^{0}$ and $K_{2}^{0}$, were eigenstates of CP with eigenvalues +1 and -1 , respectively. Since the charged pions had intrinsic parity $P_{\pi}=-1$, it was clear that only the $K_{1}^{0}$ state could decay to two charged pions, if CP was conserved, while the $K_{2}^{0}$ state would have a longer lifetime and more complex decay modes. (This argument assumes that the intrinsic parities of the $K^{0}$ and $\bar{K}^{0}$ mesons are both even.)

The suggestion of Landau was accepted for several years since it nicely restored some degree of symmetry in weak interaction processes. However, the 1964 surprising discovery ${ }^{2}$ of the decay of the long-lived neutral $K^{0}$ meson to two charged pions led to the conclusion that $\mathrm{CP}$ is violated in the weak interaction.

The observed violation of $\mathrm{CP}$ conservation turned out to be very small $(\approx 0.2 \%)$ compared with the maximal violations $(\approx 100 \%)$ of both $\mathrm{P}$ and $\mathrm{C}$ conservation separately. Indeed the very smallness of the apparent $\mathrm{CP}$ violation led to a variety of suggestions explaining it in a CP-conserving way. ${ }^{39,40}$ However, these efforts were unsuccessful and $\mathrm{CP}$ violation in weak interactions was accepted.

Another property of the weak interactions discovered in the late 1940s was their "universality." Analysis of experiments revealed that the coupling constants for both muon decay and muon capture were of the same order of magnitude as those for neutron $\beta$-decay. This led to the hypothesis of a universal weak interaction. ${ }^{41-45}$ It should be noted that in the standard $\mathrm{V}-\mathrm{A}$ theory it was envisaged that the weak interactions were mediated by massive charged bosons, $W^{+}$and $W^{-}$. Thus these weak interactions were referred to as "charge-changing" (CC) weak interactions. Subsequently it was found that the weak interaction involved in strangeness- 
conserving $(\Delta S=0)$ decays such as neutron $\beta$-decay is in fact slightly weaker than for muon decay. In addition, the weak interaction involved in strangeness-changing $(\Delta S=1)$ decays such as $\Lambda^{0} \rightarrow p^{+}+e^{-}+\bar{\nu}_{e}$ is weaker still by a significant factor.

In order to preserve the universality of the weak interaction for both hadronic and leptonic processes, Cabibbo ${ }^{46}$ (following earlier suggestions of Gell-Mann and Levy ${ }^{47}$ ) assumed that in hadronic processes the weak interaction is shared between $\Delta S=0$ and $\Delta S=1$ transition amplitudes in the ratio of $\cos \theta_{c}: \sin \theta_{c}$. This Cabibbo angle $\theta_{c}$ has a value $\approx 13^{\circ}$. In this way, Cabibbo was able to account for both neutron $\beta$-decay $(\Delta S=0)$ and $\Delta S=1$ decays such as $\Lambda^{0} \rightarrow p^{+}+e^{-}+\bar{\nu}_{e}$ employing a universal weak interaction: the transition probabilities of these decays are reduced by factors of $\cos ^{2} \theta_{c} \approx 0.95$ and $\sin ^{2} \theta_{c} \approx 0.05$, respectively, relative to pure leptonic decay processes such as muon decay, in agreement with experiment.

This so-called "Cabibbo mixing" is an integral part of the SM. In the quark model it leads to a sharing of the weak interaction between quarks with different flavors (different generations) unlike the corresponding case of leptonic processes. In order to simplify matters, the following discussion will be restricted to the first two generations of the fundamental particles of the SM, involving only the Cabibbo mixing, although the extension to three generations is straightforward. ${ }^{48}$ In the latter case, the quark mixing parameters correspond to the Cabibbo-KobayashiMaskawa (CKM) matrix elements.

Cabibbo mixing was incorporated into the quark model of hadrons by postulating that the so-called weak interaction eigenstate quarks, $d^{\prime}$ and $s^{\prime}$, form weak CC interaction isospin doublets with the $u$ and charmed $(c)$ quarks, respectively: $\left(u, d^{\prime}\right)$ and $\left(c, s^{\prime}\right)$. The weak eigenstate quarks are linear superpositions of the so-called mass eigenstate quarks $(d$ and $s)$ :

$$
d^{\prime}=d \cos \theta_{c}+s \sin \theta_{c}
$$

and

$$
s^{\prime}=-d \sin \theta_{c}+s \cos \theta_{c} .
$$

The quarks $d$ and $s$ are the quarks which participate in the electromagnetic and the strong interactions with the full allotted strengths of electric charge and color charge, respectively. The quarks $d^{\prime}$ and $s^{\prime}$ are the quarks which interact with the $u$ and $c$ quarks, respectively, with the full strength of the weak CC interaction.

\section{Parity of Charged Pions in Composite Generation Model}

The $\mathrm{GM}^{8-10}$ differs from the $\mathrm{SM}$ in that it treats quark mixing differently from the method introduced by Cabibbo ${ }^{46}$ and employed in the SM. Essentially, in the GM, the quark mixing is placed in the quark states (wave functions) rather than in the weak interactions. This allows a unified and simpler classification of both the leptons and quarks in terms of only three additive quantum numbers, each of which is conserved in all interactions. 
The three conserved additive quantum numbers of the GM: electric charge $(Q)$, particle number $(p)$ and generation quantum number $(g)$ are sufficient to describe all the observed transition amplitudes for both hadronic and leptonic processes, provided each "force" particle, mediating the various interactions, has $p=g=0$. This unified classification scheme for leptons and quarks indicated that leptons and quarks are intimately related and led to the development of a composite version of the GM: the CGM (Composite Generation Model). ${ }^{11,12}$

In the GM, physical quarks are considered to be mixed states, rather than pure quark states. Consequently, the quarks $d^{\prime}$ and $s^{\prime}$ given by Eqs. (8) and (9) in the two generation approximation are physical quarks which exist in reality, rather than the conceptual weak interaction eigenstates of the SM. The quarks $d$ and $s$ are the quarks which form weak CC interaction isospin doublets with the $u$ and $c$ quarks, respectively: $(u, d)$ and $(c, s)$, and interact with their isospin partners with the full strength of the weak CC interaction.

Thus in the GM, hadrons are considered to be composed of mixed-quark states, i.e. a proton essentially consists of two $u$-quarks and a $d^{\prime}$-quark, rather than two $u$-quarks and a $d$-quark as in the SM. Such mixed-quark states in hadrons readily account for the reduced transition probabilities relative to leptonic transition probabilities observed in weak interaction processes involving quarks, as described by the alternative quark-mixing mechanism introduced by Cabibbo.

The existence of mixed-quark states in hadrons has several consequences. ${ }^{10}$ One important consequence is that mixed-quark states may have mixed parity.

In the CGM the constituent particles of quarks are fermions, which we have called "rishons," following the introduction of such particles into an early composite model of leptons and quarks by Harari. ${ }^{49}$ If one adopts the simple convention that all rishons have positive parity, and all their antiparticles have negative parity, one finds that the $d$-quark and the $s$-quark have opposite intrinsic parity, according to the proposed structures ${ }^{11,12}$ of these quarks in the CGM: the $d$-quark consists of two rishons and one antirishon $\left(P_{d}=-1\right)$, while the $s$-quark consists of three rishons and two antirishons $\left(P_{s}=+1\right)$. The $u$-quark consists of two rishons and one antirishon so that $P_{u}=-1$, and the antiparticles of these three quarks have the corresponding opposite parities: $P_{\bar{d}}=+1, P_{\bar{s}}=-1$ and $P_{\bar{u}}=+1$.

In the CGM the $\pi^{-}$meson has, in the two generation approximation, the structure

$$
\pi^{-} \equiv\left[d^{\prime} \bar{u}\right]=[d \bar{u}] \cos \theta_{c}+[s \bar{u}] \sin \theta_{c} .
$$

We can denote the parity of the $\pi^{-}$meson by

$$
P_{\pi}=P_{d^{\prime}} P_{\bar{u}} \equiv P_{d^{\prime}},
$$

where

$$
P\left[d^{\prime}\right]=P_{d^{\prime}}\left[d^{\prime}\right]=P_{d}[d] \cos \theta_{c}+P_{s}[s] \sin \theta_{c},
$$


so that the $\pi^{-}$meson exists in a mixed-parity state: $\approx 95 \% P_{d}+5 \% P_{s}$, with $P_{d}=$ -1 and $P_{s}=+1$. ( $P_{d^{\prime}}$ is not an eigenvalue of the parity operator but is simply a short-hand notation for the linear superposition of mixed-parity eigenvalues, $P_{d}$ and $P_{s}$, in Eq. (12). The mixed-parity parameter $P_{d^{\prime}}$ may be treated analogously to an eigenvalue of a parity eigenstate, although unlike an eigenvalue, $\left[P_{d^{\prime}}\right]^{2}$ is $\neq 1$.)

Similarly, both the neutron $\left[u d^{\prime} d^{\prime}\right]$ and the proton $\left[u u d^{\prime}\right]$ exist in mixed-parity states: we have $P_{n}=P_{u} P_{d^{\prime}} P_{d^{\prime}}=-P_{d^{\prime}} P_{d^{\prime}}$ and $P_{p}=P_{u} P_{u} P_{d^{\prime}}=P_{d^{\prime}}$ for the neutron and proton parities, respectively.

We shall now demonstrate that the 1954 experiment of Chinowsky and Steinberger is compatible with the mixed-parity nature of the $\pi^{-}$meson, essentially determined by $P_{d^{\prime}}$.

For the reaction $\pi^{-}+D \rightarrow 2 n$, employed by Chinowsky and Steinberger, we require the conservation of both parity and total angular momentum.

As indicated in Sec. 2 the spins of the pion and the deuteron are known to be 0 and 1, respectively, and it is also known that the pion is captured by the deuteron from an $\mathrm{S}$ state so that the relative orbital angular momentum in the initial state is $l_{i}=0$ and consequently the total angular momentum of the initial state is $j_{i}=1$. Thus the overall parity of the initial state is given by

$$
(-1)^{l_{i}} P_{\pi} P_{D}=(-1)^{0} P_{d^{\prime}} P_{n} P_{p}=P_{d^{\prime}}\left(-P_{d^{\prime}} P_{d^{\prime}}\right) P_{d^{\prime}}=-\left[P_{d^{\prime}}\right]^{4} .
$$

The overall parity of the final state for relative orbital angular momentum $l_{f}$ is

$$
(-1)^{l_{f}} P_{n} P_{n}=(-1)^{l_{f}}\left(-P_{d^{\prime}} P_{d^{\prime}}\right)\left(-P_{d^{\prime}} P_{d^{\prime}}\right)=(-1)^{l_{f}}\left[P_{d^{\prime}}\right]^{4} .
$$

Again, as discussed in Sec. 2, since the neutrons are identical fermions, the only allowed antisymmetric state of the two neutrons with $j_{f}=1$ (conservation of total angular momentum) is a ${ }^{3} P_{1}$ state, so that $l_{f}=1$, and hence the parity of the final state is the same as the parity of the initial state, $-\left[P_{d^{\prime}}\right]^{4}$. Thus, although parity is conserved in the reaction, the mixed-parity of the $\pi^{-}$meson, $P_{d^{\prime}}$, is not determined by the experiment.

Another important consequence of the mixed-parity nature of the charged pions is that it provides a quantitative description of the decay of the long-lived $K_{2}^{0}$ meson into two charged pions: $K_{2}^{0} \rightarrow \pi^{+}+\pi^{-}$, as discovered by Christenson et al. ${ }^{2}$ without the violation of CP symmetry in the weak interaction process.

As discussed in Sec. 2, in the SM such a decay indicated the violation of CP symmetry in the weak interaction process, since the $K_{2}^{0}$ meson is considered to exist in a $\mathrm{CP}=-1$ eigenstate, while the final two charged pion state is a $\mathrm{CP}=$ +1 eigenstate. However, this conclusion depends critically upon the charged pion parity being $P_{\pi}=-1$.

In the CGM, as described in detail in Ref. 13 , the $K_{2}^{0}$ meson exists in a $\mathrm{CP}=$ -1 eigenstate but due to quark-mixing the final $\left[\pi^{+} \pi^{-}\right]$state exists in a mixed CP state, containing a very small component of $\mathrm{CP}=-1$ eigenstate. Consequently the $K_{2}^{0}$ meson may decay to two charged pions with the conservation of CP in the weak interaction process. 


\section{Conclusion}

It has been shown that the original determination of the parity of the negatively charged pion, using the capture of negatively charged pions in deuterium to form two neutrons, is not conclusive, if the pion has a complex substructure as in the CGM. In addition it has been demonstrated that this experiment is compatible with the mixed-parity nature of the $\pi^{-}$arising in the CGM.

The mixed-parities of the charged pions have previously ${ }^{13}$ been shown to provide a quantitative description of the decay of the long-lived $K_{2}^{0}$ meson into two charged pions with the conservation of CP symmetry. This suggests that perhaps CP is not violated in the $K^{0}-\bar{K}^{0}$ system and that the experiment of Christenson et al. ${ }^{2}$ unintentionally "discovered" quark-mixing in hadrons rather than CP violation in nature.

\section{Acknowledgments}

I am grateful to N. H. Fletcher, D. Robson and L. J. Tassie for helpful discussions.

\section{References}

1. T. D. Lee and C. N. Yang, Phys. Rev. 104 (1956) 254.

2. J. H. Christenson et al., Phys. Rev. Lett. 13 (1964) 138.

3. K. Gottfried and V. F. Weisskopf, Concepts of Particle Physics, Vol. 1 (Oxford University Press, New York, 1984).

4. W. Chinowsky and J. Steinberger, Phys. Rev. 95 (1954) 1561.

5. M. Gell-Mann, Phys. Lett. 8 (1964) 214.

6. G. Zweig, CERN reports 8182/TH 401 and 8419/TH 412 (1964).

7. W. Greiner and B. Müller, Gauge Theory of Weak Interactions, 4th edn. (Springer, Berlin, 2009).

8. B. A. Robson, Int. J. Mod. Phys. E 11 (2002) 555.

9. B. A. Robson, Int. J. Mod. Phys. E 13 (2004) 999.

10. P. W. Evans and B. A. Robson, Int. J. Mod. Phys. E 15 (2006) 273.

11. B. A. Robson, Int. J. Mod. Phys. E 14 (2005) 1151; [Erratum: ibid. 15 (2006) 273].

12. B. A. Robson, Int. J. Mod. Phys. E 20 (2011) 733.

13. A. D. Morrison and B. A. Robson, Int. J. Mod. Phys. E 18 (2009) 1825.

14. O. Laporte, Zeit. Phys. 23 (1924) 135.

15. E. Wigner, Zeit. Phys. 43 (1927) 624.

16. B. A. Robson, The Theory of Polarization Phenomena (Clarendon Press, Oxford, 1974).

17. B. Ferretti, Report of an International Conference on Low Temperatures and Fundamental Particles (The Physical Society, London, 1946), Vol. 1, p. 75.

18. K. Brueckner, R. Serber and K. Watson, Phys. Rev. 81 (1951) 575.

19. G. Harris, J. Orear and S. Taylor, Phys. Rev. 100 (1955) 932.

20. V. Fitch and K. Motley, Phys. Rev. 101 (1956) 496.

21. L. W. Alvarez et al., Phys. Rev. 101 (1956) 503.

22. R. H. Dalitz, Philos. Mag. 44 (1953) 1068.

23. R. H. Dalitz, Phys. Rev. 94 (1954) 1046.

24. J. Orear, G. Harris and S. Taylor, Phys. Rev. 102 (1956) 1676.

25. W. Chinowsky and J. Steinberger, Phys. Rev. 100 (1955) 1476. 
26. C. S. Wu et al., Phys. Rev. 105 (1957) 1413.

27. R. L. Garwin, L. M. Lederman and M. Weinrich, Phys. Rev. 105 (1957) 1415.

28. J. I. Friedman and V. L. Telegdi, Phys. Rev. 105 (1957) 1681.

29. R. P. Feynman and M. Gell-Mann, Phys. Rev. 109 (1958) 193.

30. E. C. G. Sudarshan and R. E. Marshak, Phys. Rev. 109 (1958) 1860.

31. G. D. Rochester and C. C. Butler, Nature 160 (1947) 855.

32. V. D. Hopper and S. Biswas, Phys. Rev. 80 (1950) 1099.

33. W. B. Fowler et al., Phys. Rev. 93 (1954) 861.

34. M. Gell-Mann, Phys. Rev. 92 (1953) 833.

35. T. Nakano and K. Nishijima, Prog. Theor. Phys. 10 (1953) 581.

36. M. Gell-Mann and A. Pais, Phys. Rev. 97 (1955) 1387.

37. F. Eisler et al., Nuovo Cimento 5 (1957) 1700.

38. L. D. Landau, JETP 32 (1957) 405.

39. P. K. Kabir, The CP Puzzle: Strange Decays of the Neutral Kaon (Academic Press, London, 1968).

40. A. Franklin, The Neglect of Experiment (Cambridge University Press, Cambridge, U.K., 1986).

41. B. Pontecorvo, Phys. Rev. 72 (1947) 246.

42. O. Klein, Nature 161 (1948) 897.

43. G. Puppi, Nuovo Cimento 5 (1948) 587.

44. G. Puppi, Nuovo Cimento 6 (1949) 194.

45. J. Tiomno and J. A. Wheeler, Rev. Mod. Phys. 21 (1949) 144.

46. N. Cabibbo, Phys. Rev. Lett. 10 (1963) 531.

47. M. Gell-Mann and M. Levy, Nuovo Cimento 16 (1960) 705.

48. M. Kobayashi and T. Maskawa, Prog. Theor. Phys. 49 (1973) 652.

49. H. Harari, Phys. Lett. B 86 (1979) 83. 